\title{
Application of Geological Risk Fine Identification Technology in Exploration Drilling
}

\author{
Baisong Tang ${ }^{1}$, Kun $\mathrm{Xu}^{1}$, Zhankui $\mathrm{Li}^{2}$, Jinting $\mathrm{Zhu}^{2}$, Rui Song ${ }^{2}$, Xiaojun Guo ${ }^{2}$ \\ ${ }^{1}$ Tianjin Branch of China National Offshore Oil Co., Ltd. Tianjin 300459, China \\ ${ }^{2} \mathrm{CNOOC}$ Ener Tech-Drlling\&Production Co. Tianjin 300452, China
}

\begin{abstract}
As exploration progresses, complex block formations and mid-deep formations have become the main target areas in Bohai oil fields. In this context, well leaks and well wall instability due to special lithology are becoming more and more likely to occur, and have become the most common complications affecting the safety of drilling operations and production timing in the Bohai Sea. Geological risk prediction can give engineering hints at the design stage, so that the engineering is in a position to prepare for the risks, but because the risk prediction given on conventional seismic profiles is relatively rough, the practical application deviates greatly and cannot be closely integrated with the actual operation. In this paper, we analyze the data of leaking wells in the Bohai exploration wells, summarize the leaking well tectonic background and stratigraphy, and divide the leak-prone tectonic zone. By extracting suitable seismic attributes such as variance and waveform, fine prediction of weak (fractured) well sections is realized, and a set of fine identification techniques is formed to Uvoid,'prevent'and plug'well leaks. dvoid,'prevent'and plug'well leakage. This technology has been applied in several exploration wells in the Bohai Sea, and it has played a good effect and is of great significance to promote.
\end{abstract}

Keywords: Bohai Bay, Geological risk, Variance, Waveform, Fine identification.

\section{Preface}

By summarizing the complex situation of exploration wells in Bohai oilfield in the past 10 years, it can be seen that the deep formation leakage has increased significantly, and the percentage of leakage in wells deeper than $3000 \mathrm{~m}$ has increased from $17 \%$ to $59 \%$ in the past 5 years. The proportion of serious leakage is increasing, and the frequency of leakage is significantly higher. If accurate prediction can be made before drilling, complex situations can be avoided by adjusting well deployment, adjusting trajectory direction, and optimizing engineering parameters for early prevention. The method used in the past was mainly to use seismic profiles to observe possible geological risks such as faults in both directions of the borehole. This kind of prediction is more rough and has a large prediction error. The fine identification technology of geological risk is currently a gap in the industry. With the integrated management mode of engineering and geology, Bohai Oilfield has developed the fine identification technology by integrating the characteristics of different specialties and digging deeper into different seismic attributes, which is widely used in engineering operations with fine prediction and high accuracy. The technology system can be excavate, upgraded and grope out with a lot of space and has very large practical benefits.

\section{Research Background}

The geological data such as seismic profiles, geological risk prediction, formation pressure calculation, stratigraphic and lithological prediction included in the well proposal and geological design are the main basis for the drilling engineering design and construction to be followed. Conventional seismic profiles can show more obvious geological data such as large faults and stratigraphic unconformities. Some subtle stratigraphic changes are difficult to be reflected from conventional profiles. With the increasing depth of wells, it is difficult to deal with complicated situations when they occur, so this limitation is especially obvious in recent years.
1) Failure to differentiate the location of a single well from a macroscopic perspective to better predict geological risk.

2) Mainly suggesting obvious faults and geological risks, with less attention to tiny fracture zones and faults.

3) The data analysis of the upper non-reservoir section is not detailed enough.

4) Fewer seismic sections over the borehole, and the data are not comprehensive enough.

Targeted research was conducted to address the limitations of conventional prediction, and finally a set of fine geological risk identification techniques applicable to the Bohai oilfield was developed to make risk prediction more scientific.

\section{Technical Thoughts}

\subsection{Macroscopic Analysis}

Through statistical data, it is found that the main leakage layer is the Paleogene, showing the distribution characteristics of "shallow in the north and deep in the south", and the fracture leakage of the tertiary strata in the Bohai Sea is closely related to the Tanlu Slip Fracture Zone.

1) Multi-phase tectonic movements are superimposed and compounded to form fracture development zones.

2) The sudden change in stratigraphic production is the location of potential fault fracture development.

3) High-angle faults (greater than $60^{\circ}$ ) have weak vertical stress effects, and the faults are not easily closed.

4) Planar crossover " $X$ " faults, stress concentration release area, fracture development and intertwined, high risk of well leakage within $300 \mathrm{~m}$ from the intersection point. 
5) The volcanic channels are filled with blocky volcanic rocks with poor stratification and fracture development inside. During the formation of the volcanic channel, its up-arching and extrusion caused the top and surrounding rocks to fracture and break up, and in the formation of a baked metamorphic zone, producing a network of fractures. The actual drilling shows that the volcanic channel and its surrounding $100 \mathrm{~m}$ area is a high risk area for well leakage.

6) Basalt and andesite have pore almond structure, and primary pores, joints, condensation shrinkage fractures and secondary micro pores are more developed. In the case of developing faults, the fractures and pores communicate with each other and form a certain accommodating space, which is a high-risk area for well leakage.

Macroscopic analysis focuses on qualitatively understanding whether a well is located in a leak-prone area and classifying the well into different levels of risk by combining seismic profile characteristics and neighboring well information.

\subsection{Fine Recognition Technology}

Conventional seismic profile (variable density seismic profile): The strength of seismic wave energy is indicated by the magnitude of the density value. Strong amplitude results in high light density and dark tones; weak amplitude results in low light density and light tones. It can reflect the fracture characterization very intuitively, but lacks detailed

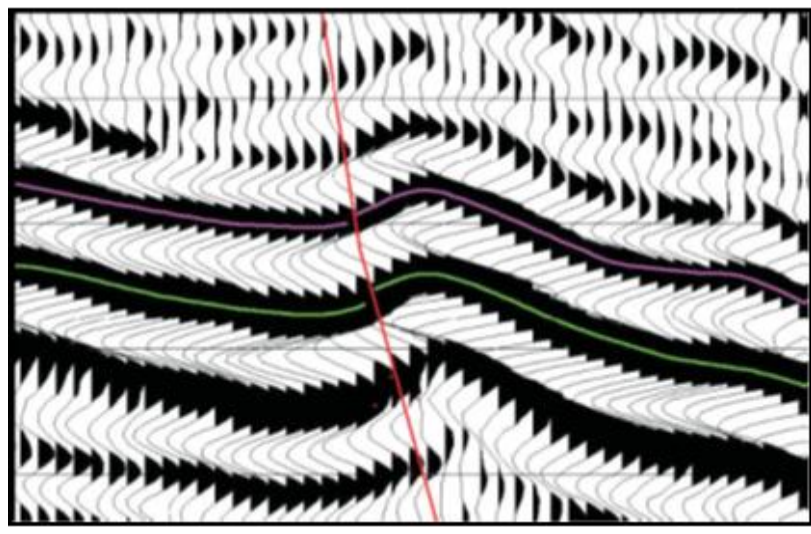

(a) Same phase axis twisting

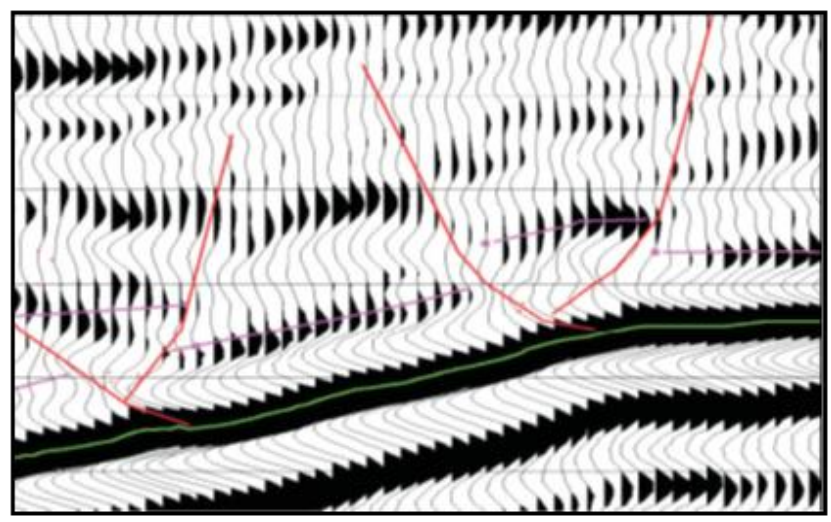

(c) Cophase axis microdislocation and dislocation

Figure 1: Seismic response characteristics of cracks on seismic reflection features

\section{Field Applications}

\subsection{Applications Situation}

information. Seismic waveform profile: The waveform of the seismic record is represented by vibration graphics, which can reflect the details of the dynamic characteristics of seismic waves (such as amplitude, frequency and phase, etc.) more comprehensively. The orthorectified results show that the larger the break distance, the easier it is to identify. When the break distance is small, the waveform profile is only slightly distorted in the homogeneous axis; as the break distance increases, the homogeneous axis distortion will be misaligned by half a phase; when the break distance continues to increase, the homogeneous axis will be significantly misaligned. In the seismic waveform profile, the fracture has some unique seismic response characteristics in the seismic reflection characteristics.

By digging deeper into the seismic information, starting from the sound wave and variance profiles, and cutting the seismic profiles by the "meters" method again, we can analyze the information hidden in the waveform data anomalies in multiple dimensions, and use the respective data features to complement and corroborate each other. The regular processed seismic profiles are mainly used for predicting the more obvious apparent geological risks, and tracing the waveform transformation to get more details, and identifying more potential geological risks through variance anomalies. The multi-faceted and precise data processing identifies many geological risks that are not easily detected, including faults, special lithologies, volcanic channels, craters and weak layers, and finally forms a prediction chart.

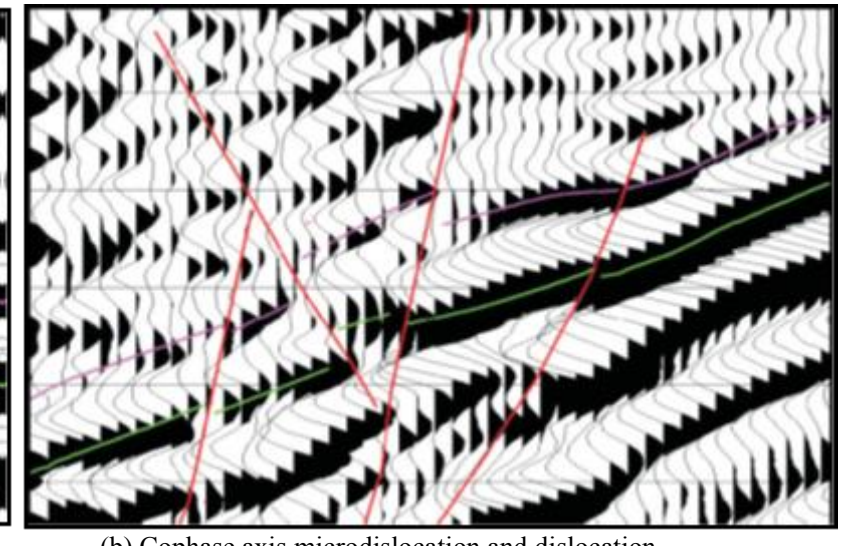

(b) Cophase axis microdislocation and dislocation

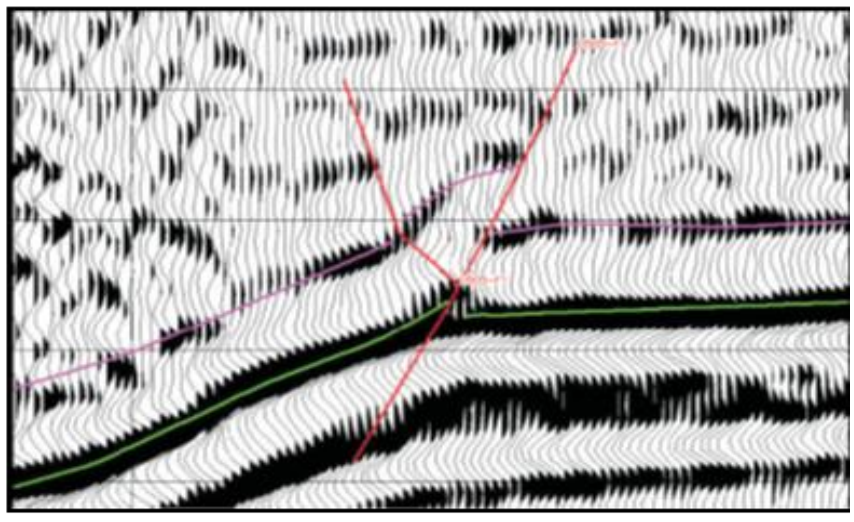

(d) Abrupt changes in the attitude of homophase axial strata

The BZ 1 well is a pre-prospect well with a design depth of $5,525 \mathrm{~m}$. The stratum encountered in the well is complex and is a key exploration well deployed in the Bohai Oilfield. No obvious geological risk was found from the conventional 
section, so the well was re-identified by fine identification technology. The "re" predicted geological risks were identified before the operation, and the risks were graded according to their characterization to guide the engineering design.

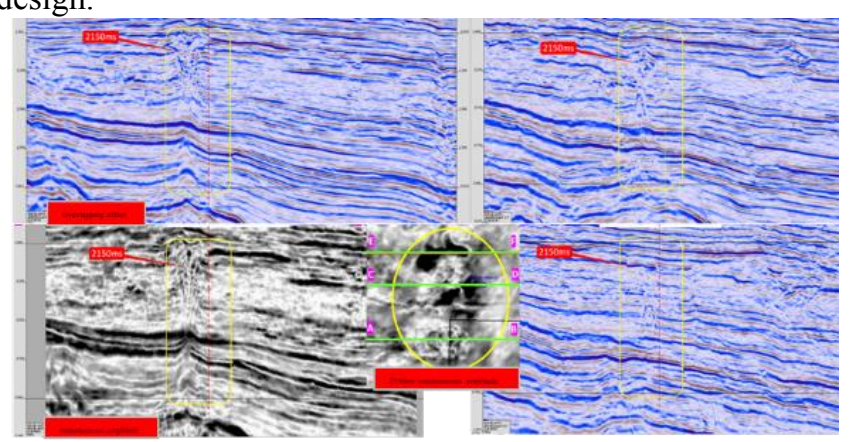

Figure 2: Seismic profile of a well in BZ

From the above figure, it can be seen that the $A B$ profile (conventional and instantaneous amplitude) can show obvious volcanic channel reflection characteristics, and combined with the EF profile and the instantaneous amplitude properties of the time slice, it can be seen that the overflow phase basalt may develop in the EF profile, so it is presumed that the overflow phase volcanic rocks develop in the vicinity of the well on the overhole $\mathrm{CD}$ profile, and the probability of developing volcanic channels in the southwest of the well is higher.

According to the risk level and well structure design principles, we will first try to avoid the more serious geological risks, but if we cannot avoid them, we will make plans and preparations, including preparing materials in advance and adjusting drilling parameters in advance to minimize the impact of geological risks.

Sixteen exploratory wells were leakage loss in 2020, with $1,430.75$ hours (59.6 days) of lost time and an average of 89.42 hours of lost time per well.

The well took targeted measures in advance in both well leaks, and a total of $75 \mathrm{~m}^{3}$ of drilling fluid was lost for 18 hours, which took only $1 / 5$ of the average processing time, resulting in a significant increase in operational timeliness and ultimately a smooth completion of drilling. The small error range of fine identification and accurate prediction also effectively shortened the non-productive time of dealing with complex situations on site because of the advance preparation.

\section{Conclusion}

(1) Macro analysis is based on familiarity with geological formations and is obtained through statistical data and analysis, and the grade it divides are of high reference value for mature blocks.

(2) Fine identification technology makes full use of waveform and variance profiles to analyze possible geological risks in detail, and gives the risk level of operating wells in combination with macro analysis, which can reasonably guide field operations.

(3) In addition to plugging, the fine identification technology of geological risk also plays a role in avoiding complex strata and adjusting trajectories.

(4) The technology system is highly replicable and can be extended to other oil fields for application, which is of great significance for promotion.

\section{References}

[1] S. K. Davlatshoev. Quality Control of Cementation Operations Performed in Country Massif in a Test Section by Seismic Well Logging[J]. Power Technology and Engineering,2020,54(2).

[2] John H Trefry,Jerry M Neff. Effects of offshore oil exploration and development in the Alaskan Beaufort Sea: A three-decade record for sediment metals $[\mathrm{J}]$. Integrated Environmental Assessment and Management,2019,15(2):

[3] Yulia A. Uvarova, ichael F. Gazley,James S. Cleverley,Aaron Baensch,David Lawie,Monica leGras. Representative, high-spatial resolution geochemistry from diamond drill fines (powders): An example from Brukunga, Adelaide, South Australia[J]. Journal of Geochemical Exploration, 016, 70.

[4] Saeed Soltani-Mohammadi, rdeshir Hezarkhani. A Simulated Annealing-Based Algorithm to Locate Additional Drillholes for Maximizing the Realistic Value of Information[J]. Natural Resources Research, 013, 2(3). 